\title{
Variations of circulating cardiac biomarkers during and after anthracycline-containing chemotherapy in breast cancer patients
}

\author{
Pierre Frères ${ }^{1,2}$, Nassim Bouznad ${ }^{3}$, Laurence Servais ${ }^{3}$, Claire Josse $^{2}$, Stéphane Wenric ${ }^{2}$, Aurélie Poncin ${ }^{1,2}$, \\ Jérôme Thiry ${ }^{2}$, Marie Moonen ${ }^{4}$, Cécile Oury ${ }^{3}$, Patrizio Lancellotti ${ }^{3,4}$, Vincent Bours ${ }^{2}$ and Guy Jerusalem ${ }^{1 *}$
}

\begin{abstract}
Background: Over time, the chance of cure after the diagnosis of breast cancer has been increasing, as a consequence of earlier diagnosis, improved diagnostic procedures and more effective treatment options. However, oncologists are concerned by the risk of long term treatment side effects, including congestive heart failure (CHF).

Methods: In this study, we evaluated innovative circulating cardiac biomarkers during and after anthracycline-based neoadjuvant chemotherapy (NAC) in breast cancer patients. Levels of cardiac-specific troponins T (cTnT), N-terminal natriuretic peptides (NT-proBNP), soluble ST2 (sST2) and 10 circulating microRNAs (miRNAs) were measured.

Results: Under chemotherapy, we observed an elevation of cTnT and NT-proBNP levels, but also the upregulation of sST2 and of 4 CHF-related miRNAs (miR-126-3p, miR-199a-3p, miR-423-5p, miR-34a-5p). The elevations of cTnT, NT-proBNP, sST2 and CHF-related miRNAs were poorly correlated, suggesting that these molecules could provide different information.

Conclusions: Circulating miRNA and SST2 are potential biomarkers of the chemotherapy-related cardiac dysfunction (CRCD). Nevertheless, further studies and long-term follow-up are needed in order to evaluate if these new markers may help to predict CRCD and to identify the patients at risk to later develop CHF.
\end{abstract}

Keywords: Biomarkers, Cardiotoxicity, Chemotherapy, Soluble ST2, microRNAs

\section{Background}

The cancer burden is a worldwide major public health problem. Fortunately, the outcome, including the cancer death rate, can be improved by earlier diagnosis and better treatment [1].

Because more patients are cured, the attention is now focusing on quality of life and long-term outcome of cancer survivors. Cardiovascular disease is the leading cause of late mortality among survivors of childhood and adolescent cancer. The risk of cardiovascular death is higher than the actual risk of cancer recurrence in many adult cancer patients in complete remission. Cancer survivors have a ten-fold higher mortality than the general population, with a fifteen-fold increased risk of

\footnotetext{
* Correspondence: g.jerusalem@chu.ulg.ac.be

${ }^{1}$ Department of Medical Oncology, University Hospital (CHU) and University of Liège, Liège, Belgium

Full list of author information is available at the end of the article
}

developing a congestive heart failure (CHF) and a tenfold increased risk of coronary artery disease [2, 3]. These patients also have a higher risk of atherosclerosis, hypertension, pericardial disease, valvular heart disease and dyslipidemia $[4,5]$. The higher cardiovascular death rate in cancer survivors is secondary to a combination of cancer treatment-related risk effects (ionizing irradiation, cytotoxic and targeted agents), familiar risk factors and health behavior. Consequently, cancer survivors need appropriate surveillance in order to early detect longterm side effects of cancer therapy, allowing appropriate treatment before the toxicity becomes irreversible $[4,5]$.

In this respect, easily accessible circulating biomarkers could be seen as highly valuable diagnostic tools for early detection of cardiotoxicity related to cancer treatments.

The cardiac-specific isoenzymes of troponins $\mathrm{T}$ and $\mathrm{I}$ (cTnT, cTnI) are released into the blood when cardiomyocytes are damaged. Troponin levels rapidly increase 
after chemotherapy and might predict late cardiac events [6]. However, troponins release is related to lysis of myocardial cells, which may be initially absent in case of type II heart damage chemotherapy. In addition, their halflife is relatively short $(2 \mathrm{~h})$ [7], requiring the collection of multiple blood samples during the treatment, which is hardly feasible during outpatient's treatments.

$\mathrm{N}$-terminal brain natriuretic peptides (NT-proBNP) are released by left ventricular cardiomyocytes in response to wall stress. This biomarker has wellestablished clinical utility in CHF. NT-proBNP elevation during chemotherapy in breast cancer patients has been related to asymptomatic decline in left ventricular ejection fraction (LVEF) [8-11]. Nevertheless, studies evaluating the predictive role of NT-proBNP in the detection of chemotherapy-related cardiac dysfunction (CRCD) gave conflicting results and the threshold of positive tests has not been determined yet [12,13].

Soluble ST2 (sST2), a member of the interleukin-1 receptor family, is a more recently discovered biomarker of cardiovascular stress. Two studies have demonstrated that high levels of sST2 may be a strong predictor of cardiovascular death in CHF patients [14, 15]. Furthermore, sST2 has a higher discrimination power than NTproBNP in CHF patients [16], and in community-based populations free of cardiovascular disease [17].

MicroRNAs (miRNAs) are approximately 22-nucleotide long RNAs that regulate gene expression by binding to and consequently silencing target messenger RNAs. They are involved in multiple biological processes including cell proliferation, differentiation and apoptosis. All cell types release miRNAs in peripheral blood under both normal and pathological conditions. Therefore, circulating miRNAs are promising biomarkers for the early and minimally invasive diagnosis of cancer and its treatment-related cellular toxicity [18-24].

The use of anthracyclines in the treatment of breast cancer is limited by dose-dependent cardiotoxicity, which eventually may lead to CHF. The aim of the present study is to evaluate the variations of innovative biomarkers during and after anthracycline-containing chemotherapy. Plasma levels of cTnT, NT-proBNP, sST2 and 10 selected miRNAs were measured in a total of 45 breast cancer patients receiving anthracyclines as part of their neoadjuvant chemotherapy (NAC). The neoadjuvant setting was selected because patients were treatment-naive, so that markers levels were not modified by other therapies such as surgery or radiotherapy.

\section{Methods}

\section{Population}

Ethics approval was obtained from the local Institutional Review Board and the Ethic Committee (Ethical Committee of the Faculty of Medicine of the University of
Liège). This prospective study was performed in compliance with the Declaration of Helsinki. Patients with treatment-naive primary breast cancer $(n=45$, median age $=49$ years, range $=26-78$ years) were recruited prospectively at $\mathrm{CHU}$ of Liège (Liège, Belgium) from 7/2011 to $9 / 2014$. All patients signed a written informed consent form. Biomarker results were not communicated to the treating physicians and consequently did not lead to any change in treatment. Patients and tumor characteristics are summarized in Table 1.

\section{Chemotherapy treatment}

All patients received NAC consisting in the sequential use of 4 courses of alkylating (cyclophosphamide) and anthracycline-based (epirubicin) chemotherapy followed by 9 to 12 weeks of tubulin-binding agent (paclitaxel) based chemotherapy. Seventeen patients suffering from HER2 amplified breast cancer received, in addition, targeted therapy (trastuzumab or lapatinib) administered concomitantly with tubulin-binding agents.

\section{Plasma collection}

Blood samples were drawn at baseline before NAC (NA1), after 2 cycles of anthracycline-containing chemotherapy (NA2), at the end of the chemotherapy 8 days before surgery (D8) and 3 months after the surgery ( $3 \mathrm{M})$, as shown in Fig. 1. Plasma was collected in $9 \mathrm{ml}$ EDTA tubes, was prepared within $1 \mathrm{~h}$ by retaining supernatant after double centrifugation at $4{ }^{\circ} \mathrm{C}(10 \mathrm{~min}$ at $815 \mathrm{~g}$ and $10 \mathrm{~min}$ at $2500 \mathrm{~g}$ ) and was stored at $-80^{\circ} \mathrm{C}$.

\section{Plasma concentration of cardiac-specific troponins T, $\mathrm{N}$-terminal brain natriuretic peptides and soluble ST2} cTnT and NT-proBNP were assessed in plasma with highly sensitive third-generation quantitative test (electrochemoluminescence method ECLIA, Roche Diagnostics, Belgium), as recommended by the manufacturer. Detection limit was $14 \mu \mathrm{g} / \mathrm{L}$ for cTnT and $400 \mathrm{ng} / \mathrm{mL}$ for NT-proBNP.

The concentrations of sST2 in the plasma were measured using a IL-1 R4/ST2 enzyme-linked immunosorbent assay (R\&D Systems, United Kingdom), with a mean minimal detectable dose of $5.1 \mathrm{pg} / \mathrm{ml}$. The inter- and intra-assay variation was $6 \%$ and $5 \%$, respectively.

\section{Selection, extraction and qRT-PCR of microRNAs}

Based on previous publications, potentially interesting miRNAs in the context of the CRCD were selected. Three groups of miRNAs were determined: i) Acute myocardial infarction (AMI)-related miRNAs, including miR-1, miR-133a, miR-133b and miR-499-5p [25]; ii) CHF-related miRNAs, including miR-208a, miR-208b, miR-126-3p, miR-199a-3p and miR-423-5p [26-28]; and iii) miR-34a-5p, which is highly upregulated after 
Table 1 Characteristics of patients and tumors (NA = not assessed, IDC = invasive ductal carcinoma, ILC = invasive lobular carcinoma)

\begin{tabular}{|c|c|}
\hline Characteristics & $\begin{array}{l}\text { Primary breast cancer } \\
\text { patients }(n=45)\end{array}$ \\
\hline Median age (range) (y) & $49(26-78)$ \\
\hline Estrogen receptor [n (\%)] & $29(64)$ \\
\hline Progesterone receptor [n (\%)] & $26(58)$ \\
\hline HER2 [n (\%)] & $17(38)$ \\
\hline Ki67 (median \pm SD) (\%) & $30 \pm 24$ \\
\hline \multicolumn{2}{|l|}{ Initial T staging [n (\%)] } \\
\hline NA & 0 \\
\hline 1 & $4(9)$ \\
\hline 2 & $23(51)$ \\
\hline 3 & $7(16)$ \\
\hline 4 & $11(24)$ \\
\hline Lymph node involvement [n (\%)] & $33(73)$ \\
\hline \multicolumn{2}{|c|}{ Tumor node metastasis (TNM) stage [n (\%)] } \\
\hline NA & 0 \\
\hline 1 & 0 \\
\hline 2 & $26(58)$ \\
\hline 3 & $19(42)$ \\
\hline \multicolumn{2}{|c|}{ Scarff-Bloom-Richardson grading system [n (\%)] } \\
\hline NA & 0 \\
\hline 1 & 0 \\
\hline 2 & $19(42)$ \\
\hline 3 & $26(58)$ \\
\hline \multicolumn{2}{|l|}{ Histologic subtype [n (\%)] } \\
\hline NA & 0 \\
\hline IDC & $42(93)$ \\
\hline ILC & $3(7)$ \\
\hline Others & 0 \\
\hline Lymphovascular invasion [n (\%)] & $9(20)$ \\
\hline \multicolumn{2}{|l|}{ Cardiovascular risk factors [n (\%)] } \\
\hline Smoking & $12(27)$ \\
\hline Type 2 diabetes & $4(9)$ \\
\hline High blood pressure & $9(20)$ \\
\hline Obesity & $7(16)$ \\
\hline Dyslipidemia & $21(47)$ \\
\hline Chronic kidney disease & $2(4)$ \\
\hline \multicolumn{2}{|c|}{ Left ventricular ejection fraction (median \pm SD) (\%) } \\
\hline Before chemotherapy & $64 \pm 11$ \\
\hline After chemotherapy & $60 \pm 29$ \\
\hline
\end{tabular}

anthracycline treatment [23] and correlated with cardiac aging and function [29].

Essential MIQE (Minimum Information for Publication of Quantitative Real-Time PCR Experiments) guidelines were followed during specimen preparation [30].
Circulating miRNAs were purified from $100 \mu \mathrm{l}$ of plasma with the miRNeasy mini kit (Qiagen, Germany) according to the manufacturer's instructions. The standard protocol was adapted on the basis of Kroh's recommendations [31]. MS2 (Roche, Belgium) was added to the samples as a carrier, cel-miR-39 and cel-miR-238 were added as spike-ins. RNA was eluted in $50 \mu \mathrm{l}$ of RNase-free water at the end of the procedure.

Reverse transcription was performed using the miRCURY LNA ${ }^{\mathrm{ma}}$ Universal RT microRNA PCR, polyadenylation and cDNA synthesis kit (Exiqon, Denmark). Quantitative PCR was performed according to the manufacturer's instructions on custom panels with the 10 selected miRNAs (Pick-\&-Mix microRNA PCR Panels, Exiqon). Controls included the reference genes described in the text, inter-plate calibrators in triplicate (Sp3) and negative controls.

All PCR reactions were performed on a LightCycler 480 Real-Time PCR System (Roche, Belgium). miRNAs were considered for analysis with a quantification cycle (Cq) value $<36$.

Data analysis

Analyses were conducted using the $2^{-\Delta \mathrm{Cq}}$ method $(\Delta \mathrm{Cq}$ $\left.=\mathrm{Cq}_{\text {sample }}-\mathrm{Cq}_{\text {reference gene }}\right)$ for each sample to obtain a normalized expression value of the miRNAs [32]. Data were then normalized using the $\Delta \mathrm{Cq}$ method as recommended by Mestdagh et al. [33]. The mean Cq of the 3 most stable miRNAs across NAC-treated patients (miR484, miR-652 and miR-148b according GeNorm analysis of previous published data) [23] was used as reference genes in addition to the cel-miR-39 spike-in.

Statistical analyses were performed with the GraphPad Prism software, version 6.00 (GraphPad Software, USA) (www.graphpad.com/scientific-software/prism/). The normal distribution of values was evaluated with the D'Agostino-Pearson omnibus and Shapiro-Wilk tests. To compare marker levels, Student's t-test (two-tailed) and non-parametric two-sided Wilcoxon or Mann-Whitney $U$ tests were used. Correlations between continuous variables were assessed with the Spearman test. Statistical significance was established as $p<0.05\left(^{(*)}, p<0.01\left(^{(* *)}\right), p<\right.$ $0.001{ }^{(* * * *)}$ or $p<0.0001{ }^{(* * * * *)}$.

\section{Results \\ Neoadjuvant chemotherapy induces high plasma level expression of cardiac-specific troponins $\mathrm{T}, \mathrm{N}$-terminal brain natriuretic peptides and soluble ST2}

cTnT were initially undetectable in most patients, with a median level under the detection limit at baseline (NA1 time point). Their levels were then increased with a 1.3fold at NA2 $(p<0.0001)$, a 2 -fold at D8 $(p<0.0001)$ and a 1.2 -fold at $3 \mathrm{M}(p<0.01)$. An elevation in cTnT levels 


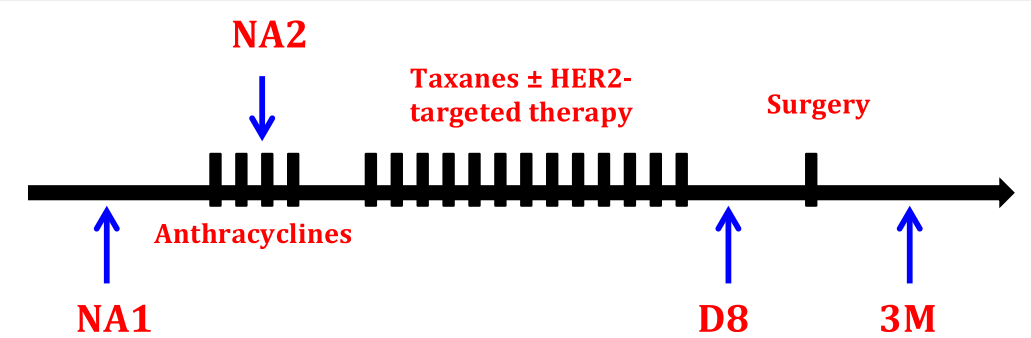

Fig. 1 Blood samples were drawn at baseline before neoadjuvant chemotherapy (NA1), after 2 cycles of anthracycline-containing chemotherapy (NA2), at the end of the chemotherapy 8 days before surgery (D8) and 3 months after the surgery ( $3 \mathrm{M}$ )

was demonstrated in $42 \%$ of patients at NA2, $73 \%$ at D8 and $47 \%$ at $3 \mathrm{M}$ (Fig. 2, Table 2).

NT-proBNP were found to be 1.4-fold elevated at NA2, 1.8-fold elevated at D8 and 1.6-fold elevated at $3 \mathrm{M}(p<$ 0.01 for each time point). The concentrations of NTproBNP were increased in $58 \%$ of patients at NA2 and $60 \%$ of patients at D8 and $3 \mathrm{M}$ (Fig. 2, Table 2).

sST2 levels were increased by 1.4 -fold in $64 \%$ of patients at NA2 $(p<0.001)$, by 1.6 -fold in $87 \%$ of patients at D8 $(p<0.0001)$ and by 1.3 -fold in $69 \%$ of patients at $3 \mathrm{M}(p<0.001)$ (Fig. 2, Table 2).

\section{Cardiac heart failure-related microRNAs plasma levels} significantly increase after neoadjuvant chemotherapy None of the AMI-related miRNAs (miR-1, miR-133a, miR-133b, miR-499-5p) were significantly deregulated during and after the NAC.

Significant increases were found regarding the levels of CHF-related miRNAs. The concentrations of miR-126$3 p$ were elevated by 1.3 -fold in $76 \%$ of patients at NA2 $(p<0.0001)$ and in $71 \%$ of patients at $3 \mathrm{M}$. This miRNA was not significantly deregulated at D8 $(p>0.05)$. miR$199 a-3 p$ levels were found elevated by 1.2 -fold in $64 \%$ of patients at NA2 $(p<0.01)$ but were not modified at D8 and $3 \mathrm{M}$ time points. miR-423-5p levels were increased by 1.6 -fold in $78 \%$ of patients at NA2 $(p<0.0001)$, by 1.5 -fold in $78 \%$ of patients at D8 $(p<0.0001)$ and by $1.3-$ fold in $73 \%$ of patients at $3 \mathrm{M}(p<0.01)$. On the other side, miR-208a and miR-208b levels were not deregulated during the NAC (Fig. 3, Table 2).

Finally, miR-34a-5p was confirmed to be sensitive to anthracycline-based chemotherapy with a 24.3 -fold increase at NA2 in $98 \%$ of patients. miR-34a-5p levels were also increased by 9 -fold at D8 and 4.1-fold at $3 \mathrm{M}$, in $89 \%$ of patients. The increase of miR-34a-5p was highly significant at each time point $(p<0.0001)$ (Fig. 3, Table 2).

\section{Correlations between cardiac biomarkers and miRNAs}

Relationships between changes in the plasma levels of the biomarkers were evaluated (see Additional file 1 for the statistical analyses). The elevation of cTnT was significantly correlated with that of NT-proBNP $(p<$ $0.01, r=0.46)$ and sST2 $(p<0.001, r=0.48)$ at D8. No correlation was found between the elevation of NTproBNP and sST2. The only correlation observed between miRNAs and the other biomarkers concerns the increase of cTnT and miR-199a-3p at D8 $(p<0.05, r=$ 0.31 ), and the rise of sST2 that is inversely correlated with that of miR-423-5p at $3 \mathrm{M}(p<0.05, r=-0.29)$.

\section{Correlations between biomarkers variations and clinical data}

All patients had normal LVEF before starting chemotherapy. A significant decline in LVEF, defined as $\geq 10 \%$ decline from baseline to $\leq 55 \%$ [34], has been noted in 7 patients (16\% of the cohort), on average 20 months after the initial ultrasound. Of these 7 patients, 6 had HER2-positive breast cancer and degraded their LVEF during or after anti-HER2 treatment. One of these patients finally developed a CHF, with an LVEF evaluated at 33\%, while she was still under adjuvant anti-HER2 treatment.

Biomarkers variations were compared between the 2 groups of patients (normal vs. decreased LVEF). No significant differences for cTnT, NT-proBNP and sST2 were found. However, the patient who developed a CHF had higher-than-average values for these 3 markers, particularly at the end of chemotherapy for sST2 (D-8 time point, 3.64 vs. $1.53 \mathrm{pg} / \mathrm{mL}, \mathrm{SD}=0.84$ ) and 3 month after surgery for NT-proBNP (3 M time point, 6.54 vs. $1.55 \mathrm{ng} / \mathrm{mL}, \mathrm{SD}=1.14)$. For miRNAs, the elevation of miR-423-5p directly after anthracyclines (NA2 time point) was significantly greater in patients with decreased LVEF ( $p=0.045,1.28$-fold, Fig. 4). The patient who developed a CRCD also had a higher elevation of miR-423-5p than the mean of other patients (2.39 vs. 1.54-fold, SD = 0.65).

\section{HER2-targeted therapies do not modify cardiac biomarkers plasma levels}

In our cohort, 17 patients with HER2-positive breast cancer received HER2-targeted therapy during the neoadjuvant setting (trastuzumab or lapatinib). HER2- 


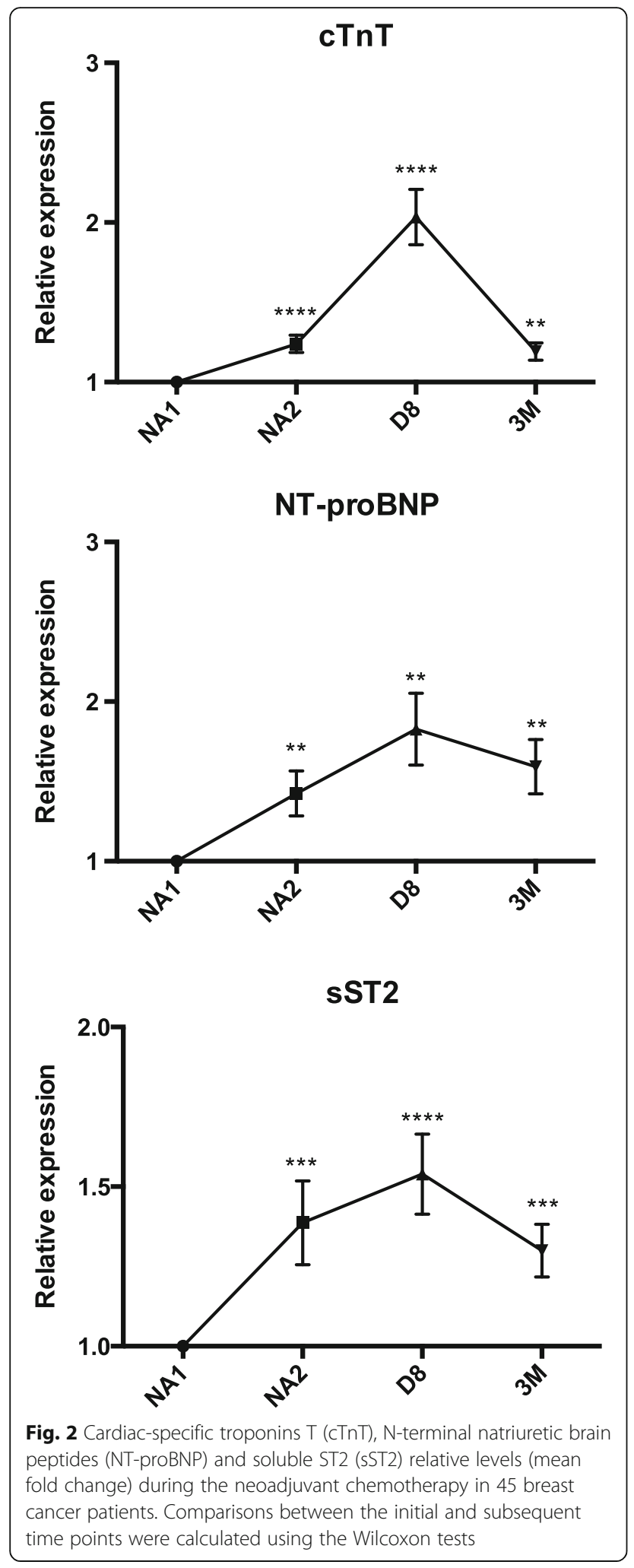

targeted therapies are associated with a modest risk of reversible cardiotoxicity, which is typically observed as an asymptomatic decrease in left ventricular function [35]. We did not observe any difference in biomarker modifications in these patients as compared with HER2negative patients (see Additional file 1).

\section{Discussion}

cTnT and NT-proBNP are both important biomarkers in heart diseases. Our study finds a significant increase in these biomarkers at the end of the NAC for breast cancer, and potentially identifies a group of patients at risk of CRCD.

Compared with cTnT and NT-proBNP, sST2 levels increase in a higher percentage of patients directly after anthracyclines-based chemotherapy (NA2 time point). As sST2 is strongly associated with CHF severity and outcome [14, 15], its blood concentration during chemotherapy may be an important predictor of long-term CRCD. Nonetheless, sST2 lacks tissue specificity and its levels could be elevated in case of breast cancer [36, 37]. In fact, Lu et al. previously reported elevated sST2 levels in the serum of breast cancer patients, with a decrease after tumor surgery [36]. All our patients experienced a complete - or at least a partial - pathologic response to the NAC, and were free of disease after 3 months. If tumor cells indeed secreted plasma sST2, we would expect a progressive decrease in its levels as the tumor responds or after the surgery. On the contrary, plasma levels of sST2 remain significantly higher, compared with baseline, after the chemotherapy and even 3 months after breast surgery (D8 and $3 \mathrm{M}$ time points, Fig. 2). Apoptotic cancer cells could also have released plasma sST2. If this was the case, we should detect a correlation between SST2 upregulation and tumor response to chemotherapy, but such a correlation was not found. Based on these results, we think that sST2 elevation is a consequence of the chemotherapy rather than a reflection of the tumor presence and/or response.

Among selected miRNAs, we demonstrated the increase of 3 miRNAs related to the diagnosis and prognosis of CHF: miR-126-3p, miR-199a-3p, miR-423-5p. Vascular endothelium-enriched miR-126 has been associated with coronary artery disease and CHF. Reduced levels were observed in the acute phase [26, 28, 38], followed by normalization after clinical improvement $[26,28]$. CRCD does not necessarily imply tissue ischemia [18] and an increase, rather than a decrease, of miR-126-3p concentrations was found after the chemotherapy. As miR-126-3p is enriched in endothelial cells and promotes blood vessels formation [39], its upregulation could be a response to cellular stress and antiangiogenic activity of the chemotherapy [40]. In the same way, miR-199a-3p levels also decreased in acute heart failure [41], while our results have shown an elevation after chemotherapy. miR-199a-3p is largely expressed in cardiomyocytes and its myocardial levels are upregulated in hypertrophic hearts [42], which is 
Table 2 Percentage of significant increase in markers levels at each time point of the chemotherapy treatment

\begin{tabular}{|c|c|c|c|c|c|c|c|}
\hline Time points & cTnT & NT-proBNP & sST2 & miR-126-3p & miR-199a-3p & miR-423-5p & miR-34a-5p \\
\hline NA2 & $42 \%$ & $58 \%$ & $64 \%$ & $76 \%$ & $64 \%$ & $78 \%$ & $98 \%$ \\
\hline D8 & $73 \%$ & $60 \%$ & $87 \%$ & - & - & $78 \%$ & $89 \%$ \\
\hline $3 M$ & $47 \%$ & $60 \%$ & $69 \%$ & $71 \%$ & - & $73 \%$ & $89 \%$ \\
\hline
\end{tabular}

sometimes observed in CRCD [18]. After myocardial infarction in mice, miR-199a promotes cardiomyocytes regeneration and recovery of cardiac functional parameters [43]. Cardiomyocytes could therefore release miR199a-3p under the effect of the chemotherapy to play a cardio-protective role, though this hypothesis requires further explorations. Plasma levels of miR-423-5p were highly increased immediately after anthracyclines (NA2 time point), especially in patients with decreased LVEF (Fig. 4). Two recent studies have unveiled that miR-423$5 p$ was enriched in the blood of patients suffering from CHF, with a high diagnosis power and a significant correlation with $\mathrm{CHF}$ prognostic markers [27, 44]. In another study, circulating miR-423-5p levels were able to predict long-term mortality of CHF patients [45]. Based on these reports and our findings, we believe that the diagnostic, prognostic and predictive roles of miR423-5p in CRCD should be further explored.

Circulating miR-34a-5p was also studied for multiple reasons. Boon et al. demonstrated that miR-34a was involved in the alteration of cardiac contractile function after AMI, by inducing telomere attrition [29]. Desai et al. evaluated miR-34a expression in myocardial tissues of mouse exposed to increasing doses of doxorubicin. Unlike troponins, upregulation of miR-34a was an early event inside the cardiac tissue and did not involve prior necrosis of cardiomyocytes [20]. Our study indicates a strong increase in miR-34a plasma levels immediately after anthracycline-based chemotherapy, followed by a gradual decrease. The tumor suppressor p53 is known to promote both growth arrest and apoptosis upon DNA damage [46]. miR-34a is directly induced by p53 to exert anti-tumor functions [47]. This might explain the miR34a upregulation observed after anthracyclines, which cause DNA breaks and p53 activation [48]. The subsequent tubulin-binding agent based chemotherapy does not act via DNA breaks but by the disruption of microtubule function. Plasma miR-34a increase could therefore be specific of anthracyclines chemotherapy, explaining the gradual decrease of miR-34a levels after

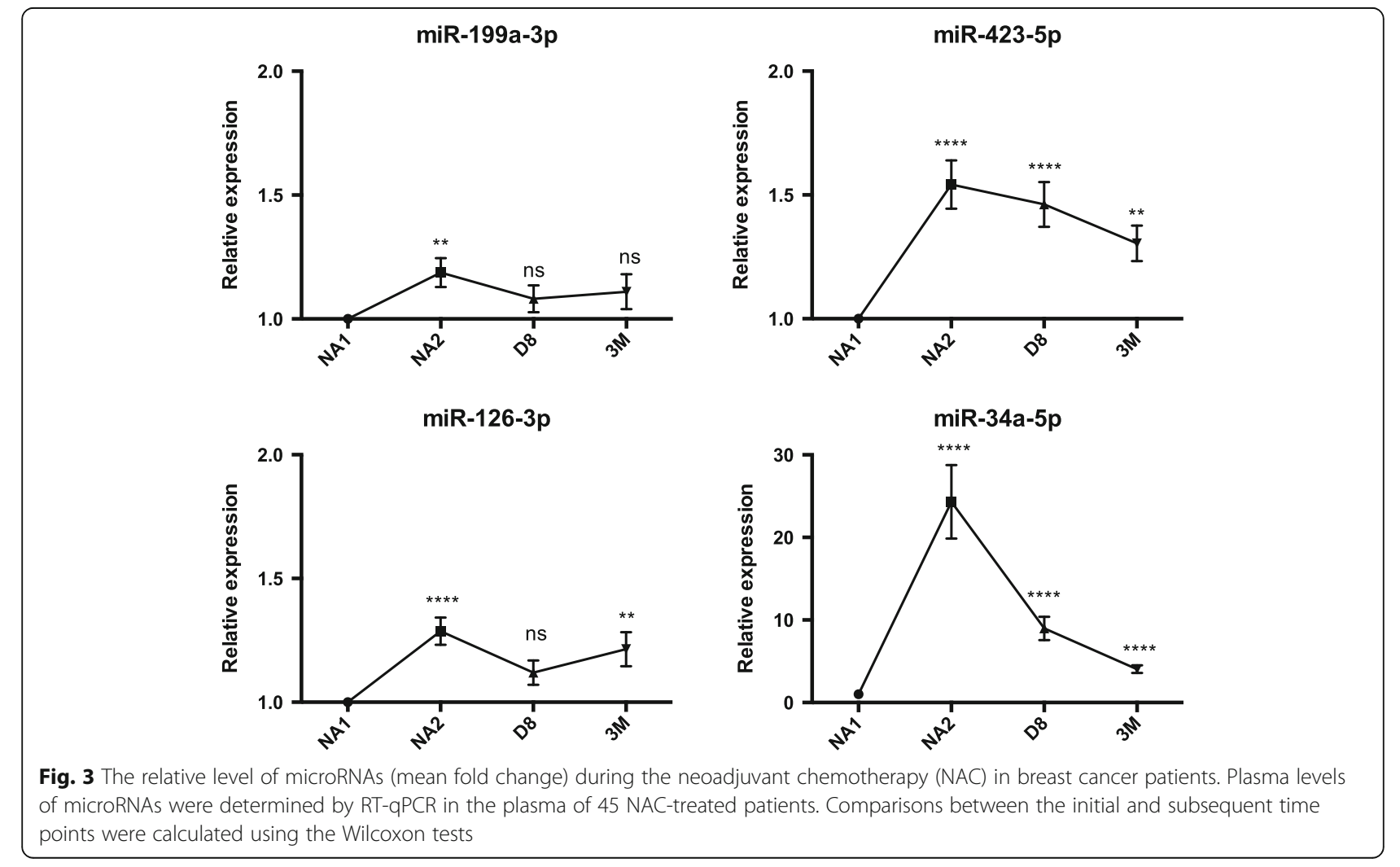




\section{miR-423-5p elevation after anthracyclines}

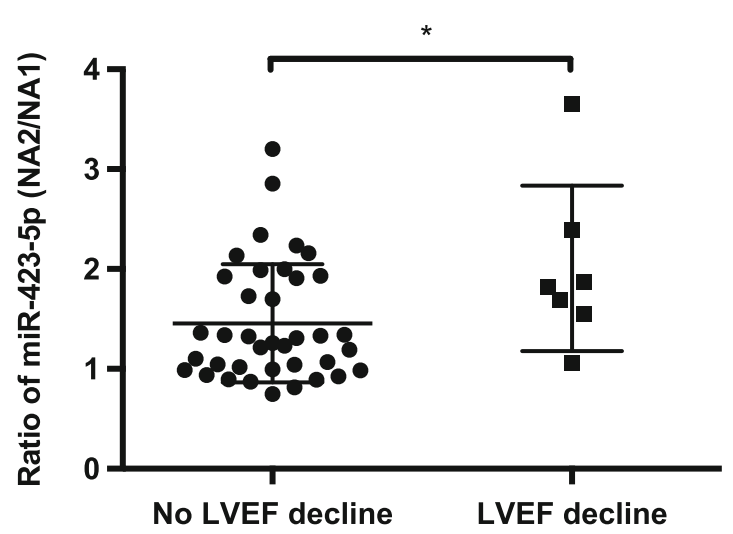

Fig. 4 Comparison of circulating miR-423-5p elevation directly after anthracyclines between patients with $(n=7)$ or without $(n=38)$ LVEF decline. Expression was determined by RT-qPCR at NA1 and NA2 time points. Comparisons between the 2 groups were calculated using the Mann-Whitney $U$ test. The data are expressed as the mean \pm SEM

this part of the treatment. The concentration of miR34a-5p may help for the identification of patients at risk of developing CRCD, however, the threshold of a positive test is still unknown. One problem may be the significant elevation in a high percentage of patients although most of them will not develop any cardiotoxicity. Furthermore, miR-34a is broadly expressed in normal tissues and this miRNA may thus lack specificity. The increase of miR-34a levels after anthracyclines was not correlated with the increase of troponins. This observation is in contradiction with our previous study that was performed on a smaller number of patients [23], probably because of the limited statistical power. Globally, the elevation of the other markers (cTnT, NTproBNP, sST2) was poorly correlated with that of CHFrelated miRNAs, which could imply that these molecules provide distinct information about CRCD.

Interpretation of our results is limited for several reasons. Firstly, the small number of patients, with only one who developed a clinical CRCD, does not allow definitive conclusions to be drawn. In addition, majority of patients experienced an asymptomatic decline in LVEF in the context of anti-HER2 therapy, and we know that the cardiotoxicity mechanism is different from that of anthracyclines [18]. As cardiomyopathy is a late side effect of the chemotherapy, a long-term follow-up is required. The biomarkers we identified (sST2, miR-1263p, miR-199a-3p, miR-423-5p and miR-34a-5p) should therefore be studied in a larger prospective trial with regular and prolonged cardiac monitoring. These biomarkers may be useful either as a predictive marker of cardiotoxicity at the time of treatment or as a new tool for the early identification of late side effects before the patients have clinical symptoms. Importantly, appropriate treatment of CRCD may prevent irreversible consequences if initiated early.

\section{Conclusion}

We identified sST2, miR-126-3p, miR-199a-3p, miR423-5p and miR-34a-5p as innovative biomarkers for potential early and sensitive detection of the cardiomyopathy associated with anthracycline-based breast cancer chemotherapy.

\section{Additional file}

Additional file 1: Statistical analyses. (A) Correlations Spearman tests between the fold change of cTnT, NT-proBNP, sST2, miR-126-3p, miR199a-3p, miR-423-5p and miR-34a-5p at different time point of the neoadjuvant chemotherapy in 45 breast cancer patients. (B) Comparison between the increase of the markers at the end of the chemotherapy (NA1 vs. D8 and NA1 vs. 3 M) in patients treated $(n=17)$ or not $(n=28)$ with HER2 targeted therapy, using Mann-Whitney tests. (XLSX 50 kb)

\section{Abbreviations}

AMI: Acute myocardial infarction; CHF: Congestive heart failure; CRCD: Chemotherapy-related cardiac dysfunction; CTnT: Cardiac-specific troponins T; LVEF: Left Ventricular Ejection Fraction; MIQUE: Minimum Information for Publication of Quantitative Real-Time PCR Experiments; miRNAs: microRNAs; NAC: Neoadjuvant chemotherapy; NT-proBNP: Nterminal brain natriuretic peptides; SD: Standard deviation; SEM: Standard error of the mean; sST2: Soluble ST2

\section{Acknowledgments}

We would like to thank Olivier Dengis, Corinne Fasquelle, Tiberio Sticca, Sonia El Guendi, Bouchra Boujemla, the GIGA-imagery-platform, the GIGAimmunohistology-platform, the team of medical oncologists and the Biobank of $\mathrm{CHU}$ de Liège.

\section{Funding}

PF is a F.R.S.-FNRS PhD fellow. LS and NB had a Televie fellowship. CO is a Senior Research Associate at the F.R.S.-FNRS. This work was supported by the French Community of Belgium; the Belgian Funds for Scientific Research (F.R.S.-FNRS); the F.R.S.-FNRS-Televie; the F.I.R.S (University Hospital of Liège) and the Région Wallone (Secance, BRAMIR).

\section{Availability of data and materials}

The datasets used and analysed during the current study are available from the corresponding author on reasonable request.

\section{Authors' contributions}

PF, NB, LS, SW, AP, and JT carried out the molecular genetic studies, participated in the design of the study and statistical analyses, and drafted the manuscript. CJ, MM, CO, and PL participated in the design of the study and the writing of the manuscript. PL, VB and GJ conceived the study, and participated in its design and coordination and helped to draft the manuscript. All authors read and approved the final manuscript.

Ethics approval and consent to participate

The ethics committee of the University Hospital of Liège approved this study. All patients signed a written informed consent form.

Consent for publication

Not applicable.

Competing interests

The authors declare that they have no competing interests. 


\section{Publisher's Note}

Springer Nature remains neutral with regard to jurisdictional claims in published maps and institutional affiliations.

\section{Author details}

'Department of Medical Oncology, University Hospital (CHU) and University of Liège, Liège, Belgium. 'Laboratory of Human Genetics, GIGA Research, University Hospital (CHU) and University of Liège, Liège, Belgium. ${ }^{3} \mathrm{GIGA}$ Cardiovascular Sciences, Department of Cardiology, Heart Valve Clinic, University Hospital (CHU) and University of Liège, Liège, Belgium. ${ }^{4}$ Gruppo Villa Maria Care and Research, Anthea Hospital, Bari, Italy.

Received: 3 November 2016 Accepted: 22 January 2018

Published online: 29 January 2018

\section{References}

1. Jemal A, Bray F, Center MM, Ferlay J, Ward E, Forman D. Global cancer statistics. CA Cancer J Clin. 2011;61(2):69-90.

2. Oeffinger KC, Mertens AC, Sklar CA, Kawashima T, Hudson MM, Meadows AT, et al. Chronic health conditions in adult survivors of childhood cancer. N Engl J Med. 2006;355(15):1572-82

3. Mertens AC, Yasui Y, Neglia JP, Potter JD, Nesbit ME Jr, Ruccione K, et al. Late mortality experience in five-year survivors of childhood and adolescent cancer: the childhood cancer survivor study. J Clin Oncol. 2001:19:3163-72.

4. Diller L, Chow EJ, Gurney JG, Hudson MM, Kadin-Lottick NS, Kawashima TI, et al. Chronic disease in the childhood cancer survivor study cohort: a review of published findings. J Clin Oncol. 2009;27:2339-55.

5. Carver JR, Shapiro CL, Ng A, Jacobs L, Schwartz C, Virgo KS, et al. American Society of Clinical Oncology clinical evidence review on the ongoing Care of Adult Cancer Survivors: cardiac and pulmonary late effects. J Clin Oncol. 2007:25:3991-4008.

6. Cardinale D, Sandri MT, Colombo A, Colombo N, Boeri M, Lamantia G, et al. Prognostic value of troponin I in cardiac risk stratification of cancer patients undergoing high-dose chemotherapy. Circulation. 2004;109:2749-54

7. Gerhardt W, Katus H, Ravkilde J, Hamm C, Jørgensen PJ, Peheim E, et al. Stroponin $\mathrm{T}$ in suspected ischemic myocardial injury compared with mass and catalytic concentrations of S-creatine kinase isoenzyme MB. Clin Chem. 1991;37:1405-11.

8. Cil T, Kaplan AM, Altintas A, Akin AM, Alan S, Isikdogan A. Use of N-terminal pro-brain natriuretic peptide to assess left ventricular function after adjuvant doxorubicin therapy in early breast cancer patients: a prospective series. Clin Drug Investig. 2009;29(2):131-7.

9. Kittiwarawut A, Vorasettakarnkij Y, Tanasanvimon S, Manasnayakorn S, Sriuranpong V. Serum NT-proBNP in the early detection of doxorubicininduced cardiac dysfunction. Asia Pac J Clin Oncol. 2013;9(2):155-61.

10. Ky B, Putt M, Sawaya H, French B, Januzzi JL Jr, Sebag IA, et al. Early increases in multiple biomarkers predict subsequent cardiotoxicity in patients with breast cancer treated with doxorubicin, taxanes, and trastuzumab. J Am Coll Cardiol. 2014;63(8):809-16.

11. De luliis F, Salerno G, Taglieri L, De Biase L, Lanza R, Cardelli P, Scarpa S. Serum biomarkers evaluation to predict chemotherapy-induced cardiotoxicity in breast cancer patients. Tumour Biol. 2016;37(3):3379-87.

12. Vogelsang TW, Jensen RJ, Hesse B, Kjær A. BNP cannot replace gated equilibrium radionuclide ventriculography in monitoring of anthracyclineinduced cardiotoxity. Int J Cardiol. 2008:124:193-7.

13. Daugaard G, Lassen U, Bie P, Pedersen EB, Jensen $K T$, Abildgaard $U$, et al. Natriuretic peptides in the monitoring of anthracycline induced reduction in left ventricular ejection fraction. Eur J Heart Fail. 2005;7:87-93.

14. Weinberg EO, Shimpo M, Hurwitz S, Tominaga S-I, Rouleau J-L, Lee RT. Identification of serum soluble ST2 receptor as a novel heart failure biomarker. Circulation. 2003;107:721-6.

15. Pascual-Figal DA, Ordoñez-Llanos J, Tornel PL, Vázquez R, Puig T, Valdés M et al. Soluble ST2 for predicting sudden cardiac death in patients with chronic heart failure and left ventricular systolic dysfunction. J Am Coll Cardiol. 2009;54(23):2174-9.

16. Gruson D, Lepoutre T, Ahn SA, Rousseau MF. Increased soluble ST2 is a stronger predictor of long-term cardiovascular death than natriuretic peptides in heart failure patients with reduced ejection fraction. Int J Cardiol. 2014;172(1):e250-2.

17. Coglianese EE, Larson MG, Vasan RS, Ho JE, Ghorbani A, McCabe EL, et al. Distribution and clinical correlates of the interleukin receptor family member soluble ST2 in the Framingham Heart Study. Clin Chem. 2012;58: 1673-81.

18. Sandhu H, Maddock H. Molecular basis of cancer-therapy-induced cardiotoxicity: introducing microRNA biomarkers for early assessment of subclinical myocardial injury. Clin Sci. 2014;126:377-400.

19. Horie T, Ono K, Nishi H, Nagao K, Kinoshita M, Watanabe S, et al. Acute doxorubicin cardiotoxicity is associated with miR-146a-induced inhibition of the neuregulin-ErbB pathway. Cardiovasc Res. 2010;87:656-64.

20. Desai VG, C Kwekel J, Vijay V, Moland CL, Herman EH, Lee T, et al. Early biomarkers of doxorubicin-induced heart injury in a mouse model. Toxicol Appl Pharmacol. 2014;281:221-9.

21. Nishimura $Y$, Kondo C, Morikawa Y, Tonomura $Y$, Torii M, Yamate J, Uehara T. Plasma miR-208 as a useful biomarker for drug-induced cardiotoxicity in rats. J Appl Toxicol. 2015;35:173-80

22. Vacchi-Suzzi C, Bauer Y, Berridge BR, Bongiovanni S, Gerrish K, Hamadeh HK, et al. Perturbation of microRNAs in rat heart during chronic doxorubicin treatment. PLoS One. 2012;7:e40395.

23. Frères $P$, Josse $C$, Bovy $N$, Boukerroucha $M$, Struman I, Bours $V$, Jerusalem $G$. Neoadjuvant chemotherapy in breast cancer patients induces miR-34a and miR-122 expression. J Cell Physiol. 2015;230:473-81.

24. Frères $P$, Wenric $S$, Boukerroucha M, Fasquelle $C$, Thiry J, Bovy N, et al. Circulating microRNA-based screening tool for breast cancer. Oncotarget. 2016;7:5416-28

25. D'Alessandra Y, Devanna P, Limana F, Straino S, Di Carlo A, Brambilla PG, et al. Circulating microRNAs are new and sensitive biomarkers of myocardial infarction. Eur Heart J. 2010;31:2765-73.

26. Akat KM, Moore-McGriff D, Morozov P, Brown M, Gogakos T, Da Rosa JC, et al. Comparative RNA-sequencing analysis of myocardial and circulating small RNAs in human heart failure and their utility as biomarkers. PNAS. 2014:111:11151-6.

27. Tijsen AJ, Creemers EE, Moerland PD, de Windt LJ, van der Wal AC, Kok WE, Pinto YM. MiR423-5p as a circulating biomarker for heart failure. Circ Res. 2010;106:1035-9.

28. Fukushima Y, Nakanishi M, Nonogi H, Goto Y, Iwai N. Assessment of plasma miRNAs in congestive heart failure. Circ J. 2011;75:336-40.

29. Boon RA, lekushi $K$, Lechner $S$, Seeger $T$, Fischer A, Heydt $S$, et al. MicroRNA34a regulates cardiac ageing and function. Nature. 2013;495:107-10.

30. Bustin SA, Benes V, Garson JA, Hellemans J, Huggett J, Kubista M, et al. The MIQE guidelines: minimum information for publication of quantitative realtime PCR experiments. Clin Chem. 2009:55(4):611-22.

31. Kroh EM, Parkin RK, Mitchell PS, Tewari M. Analysis of circulating microRNA biomarkers in plasma and serum using quantitative reverse transcriptionPCR (qRT-PCR). Methods. 2010;50(4):298-301.

32. Schmittgen TD, Livak KJ. Analyzing real-time PCR data by the comparative CT method. Nat Protoc. 2008:3:1101-8.

33. Mestdagh $P$, Van Vlierberghe $P$, De Weer A, Muth D, Westermann F, Speleman F, Vandesompele J. A novel and universal method for microRNA RT-qPCR data normalization. Genome Biol. 2009;10(6):R64.

34. Ganz WI, Sridhar KS, Forness TJ. Detection of early anthracycline cardiotoxicity by monitoring the peak filling rate. Am J Clin Oncol. 1993; 16(2):109-12.

35. Ewer MS, Vooletich MT, Durand JB, Woods ML, Davis JR, Valero V, Lenihan DJ. Reversibility of trastuzumab-related cardiotoxicity: new insights based on clinical course and response to medical treatment. J Clin Oncol. 2005;23(31): 7820-6.

36. Lu DP, Zhou XY, Yao LT, Liu CG, Ma W, Jin F, Wu YF. Serum soluble ST2 is associated with ER-positive breast cancer. BMC Cancer. 2014;14:198.

37. Gillibert-Duplantier J, Duthey B, Sisirak V, Salaün D, Gargi T, Trédan O, et al. Gene expression profiling identifies sST2 as an effector of ErbB2-driven breast carcinoma cell motility, associated with metastasis. Oncogene. 2012; 31:3516-24.

38. Fichtlscherer S, De Rosa S, Fox H, Schwietz T, Fischer A, Liebetrau C, et al. Circulating microRNAs in patients with coronary artery disease novelty and significance. Circ Res. 2010;107:677-84.

39. Wang S, Aurora AB, Johnson BA, Qi X, McAnally J, Hill JA, et al. The endothelial-specific microRNA miR-126 governs vascular integrity and angiogenesis. Dev Cell. 2008;15:261-71.

40. Luengo-Gil G, González-Billalabeitia E, Chaves-Benito A, Martínez EG, Garre EG, Vicente V, la Peña de FA. Effects of conventional neoadjuvant chemotherapy for breast cancer on tumor angiogenesis. Breast Cancer Res Treat. 2015;151:577-87. 
41. Ovchinnikova ES, Schmitter D, Vegter EL, Maaten ter JM, Valente MAE, Liu LCY, et al. Signature of circulating microRNAs in patients with acute heart failure. Eur J Heart Fail. 2016;18:414-23.

42. Song XW, Li Q, Lin L, Wang XC, Li DF, Wang GK, et al. MicroRNAs are dynamically regulated in hypertrophic hearts, and miR-199a is essential for the maintenance of cell size in cardiomyocytes. J Cell Physiol. 2010; 225:437-43.

43. Eulalio A, Mano M, Ferro MD, Zentilin L, Sinagra G, Zacchigna S, Giacca M. Functional screening identifies miRNAs inducing cardiac regeneration. Nature. 2012;492:376-81.

44. Goren Y, Kushnir M, Zafrir B, Tabak S, Lewis BS, Amir O. Serum levels of microRNAs in patients with heart failure. Eur J Heart Fail. 2012;14:147-54.

45. Seronde MF, Vausort M, Gayat E, Goretti E, Ng LL, Squire IB, et al. Circulating microRNAs and outcome in patients with acute heart failure. PLoS One. 2015:10:e0142237.

46. Boominathan L. The tumor suppressors p53, p63, and p73 are regulators of microRNA processing complex. PLoS One. 2010;5:e10615.

47. He L, He X, Lim LP, de Stanchina E, Xuan Z, Liang Y, et al. A microRNA component of the p53 tumour suppressor network. Nature. 2007;447: $1130-4$.

48. Lowe SW, Bodis S, McClatchey A, Remington L, Ruley HE, Fisher DE, et al. p53 status and the efficacy of cancer therapy in vivo. Science. 1994; 266(5186):807-10.

\section{Submit your next manuscript to BioMed Central and we will help you at every step:}

- We accept pre-submission inquiries

- Our selector tool helps you to find the most relevant journal

- We provide round the clock customer support

- Convenient online submission

- Thorough peer review

- Inclusion in PubMed and all major indexing services

- Maximum visibility for your research

Submit your manuscript at www.biomedcentral.com/submit
Biomed Central 